\title{
Sudden Cardiac Arrest (SCA)
}

- Clinical Manifestations

- Suspect in ANY athlete who collapses or loses consciousness

- Myoclonic jerks or seizure-like activity is common after collapse of SCA and should not be mistaken for seizure

- New CPR guidelines recommend CAB (circulation, airway, breathing)

- Chest compressions more important than breaths

- CPR should be started immediately if no pulse is felt

- 30 compressions, followed by two rescue breaths

- Minimize any time without compressions

- In young healthy athletes, SCA is often related to arrhythmia

- Apply AED as soon as possible - the goal is 3-5 min from time of collapse to time of first shock

- Have someone call EMS

\section{Reference}

Casa DJ, Guskiewicz KM, Anderson SA, Courson RW, Heck JF, Jimenez CC, McDermott BP, Miller MG, Stearns RL, Swartz EE, Walsh KM. National Athletic Trainers' Association position statement: preventing sudden death in sports. J Athl Train. 2012;47(1):96-118. 